\title{
DZN2 Automatic Soil Moisture Observation System Based On GPRS Transmission
}

\author{
Xue longqin ${ }^{1,2, a}$, Ye Linmao ${ }^{1,2, b}$ \\ ${ }^{1}$ Institute Of Meteorology Of Henan Province, Henan, ZhengZhou, 450003, China \\ ${ }^{2}$ CMA Key Laboratory of Agrometeorological Ensuring and Applied Technique, Zhengzhou 450003, \\ China \\ Asnowbell66@sohu.com, byjx819@163.com
}

Keywords: Soil Moisture, GPRS, Automatic Observation, DZN2

\begin{abstract}
This article introduces the system of DZN2 automatic soil moisture observation and it's application in china. The system is mainly made up of automatic collecting device, wireless data transmission system and intelligent data analysis software, and it realizes the automatic collecting for soil moisture, remote wireless transmission, real-time analysis, automatic monitoring and decision support. Meanwhile, the installation of instrument is simple, the maintenance is convenient, the monitoring for soil moisture is continuous and the data precision is high. By these rich and practical functions, business service personnel can quickly analyze the variation trend of soil moisture content in the local area, make a scientific forecast of droughts, and provide technology support for decision-making service of government based on the real-timely collected soil moisture data.
\end{abstract}

\section{Introduction}

China is a country with vast territory, complicated geographic environment and a variety of frequent disastrous weather among which drought is the most widespread and severest. According to statistics, meteorological disasters make up 70\% of natural disasters, while droughts account for approximately $50 \%$ of meteorological disasters [1]. Droughts bring the most serious impact on agricultural production. In order to respond to the drought-relief work in the new era, Henan Institute of Meteorological Sciences and China Electronics Group-27 ${ }^{\text {th }}$ Research Institute have jointly designed, researched and developed a automatic soil moisture observation system based on GPRS transmission that can monitors and collects the real-timely soil moisture data quickly and accurately, which not only enhances the time effect of observation,eliminates the manual reading error and relieves the labor intensity of staff in the meteorological stations, but also effectively improves the level of drought monitoring and defense service[2]. In accordance with the principles of simultaneous development of demand pull and business service, meteorological departments have established more than 1000 sets of DZN2 automatic soil moisture observation systems in 12 provinces, municipalities and districts all over the country since 2009, forming an effective soil moisture and drought monitoring network in various regions.

\section{Overall Architecture and Functions of System}

DZN2 automatic soil moisture observation system is mainly made up of automatic collecting device, wireless data transmission system and intelligent data analysis software, and it realizes the automatic collecting for soil moisture, remote wireless transmission, real-time analysis, automatic monitoring and decision support. By these rich and practical functions, business service personnel can quickly analyze the variation trend of soil moisture content in the local area, make a scientific forecast of droughts, and provide technology support for decision-making service of government based on the real-timely collected soil moisture data.

\section{Design of System Hardware}

Hardware of DZN2 automatic soil moisture observation system is mainly installed in fields to 
monitor the soil moisture content of farmlands. In order to meet the need of field installation and application requirements and enable the equipment to operate stably for a long-term, the design of system hardware takes high precision, low power consumption, anti-lighting impulse, anti-moisture, easy installation and maintenance and other factors into account. System hardware includes FDR soil moisture sensor, collector and solar power-supply system.

Software of sensor and collector adopts Keil C51 design, which not only has features of general high-level language, but also can directly operate the hardware of SCM system with a stronger expression and computing capability. In addition, it has an efficient code generation and supports float-point number calculation as well as other features.

\subsection{Soil Moisture Sensor}

Soil moisture sensor is designed on the basis of capacitive transducer and embedded SCM technology. Inside the device is a single-pole multi-section sensor. Each layer of the sensor is composed of two cooper rings, and the moisture variation around sensor results in the medium change of ring capacitor, thus leading to the frequency variation of LC oscillator. The sensor converts the high-frequency signal and then outputs to SCM, which calculates the current volumetric water content of soil moisture based on the established mathematical models and hydrological constants of local soil. Outside the device is a sleeve made of PVC materials, which can prevent water or other fluids from disturbing the internal components and influencing the observation of soil moisture. For the sake of anti-moisture, desiccant is also placed in the sensor.

It is stipulated in Automatic Soil Moisture Observation Standard-Interim by China Meteorological Administration that the installation depth and layer of sensor shall be determined on demand. In the agro-meteorological observation, there are generally 8 layers, namely, 0-10, 10-20, $20-30,30-40,40-50,50-60,70-80,90-100 \mathrm{~cm}$, and the layer of sensor can be adjusted according to observation requirements[3].The currently installed DZN2 automatic soil moisture observation systems are mostly of $1 \mathrm{~m}$ deep 8-layer structure. In some mountainous areas, the measured depth can't reach $1 \mathrm{~m}$ due to the thin soil layer; therefore, the observation depth is generally subject to that of soil parent material horizon.

DZN2 soil moisture sensor is installed with special tools, without digging holes or destroying soil layer structure. What is needed in maintenance is only to pull out the sensor form the sleeve, hence simple to operate and convenient for instrument calibration.

\subsection{Soil Moisture Collector}

Data collector is designed on the basis of single chip microprocessor technology and can send instructions to moisture sensor on a real-time and regular basis as well collect soil moisture data to store. With the mass memory expansion technology, the collector can store two-month punctual volumetric water content of soil moisture, and the data memory inside the collector also has the function of power-down save. The collector supports two types of transmission modes: regular automatic transmission and terminal command responding transmission. Normally, the collector transmits soil moisture observation data once per hour; in case of manual intervention, the collector can also respond to terminal commands at any time and conduct date collection and transmission. The communication between the collector and the soil moisture sensor is realized through RS485 bussing technology, which not only has a strong expansibility but also specially reinforces the anti-lighting design, ensuring the stable operation of equipment and the reliability of data transmission. The communication between the collector and the central station server is realized via wireless communication interface and the data is transmitted via GPRS network. In order to ensure the time synchronization inside the system, the collector not only provides high-precision clock, but also regularly calibrates time with the server terminal every day.

\subsection{Solar Power-supply System}

The hardware of automatic soil moisture observation system is mostly installed in fields and has no alternating current power supply, so this system is designed with a solar power-supply system, which is mainly composed of solar panel, storage battery, charge and power supply controller. Its major working modes are like this: when power is supplied by solar energy, the charge controller supplies power for the system and charges the storage battery; when there is no solar power supply, 
the storage battery supplies power for the system; and when solar energy recovers power supply, the charge controller charges the storage battery until it is full. The solar charge controller is used for automatic charge for maintenance-free lead-acid batteries. It adopts three-stage charging modes with temperature compensation, namely: strong charge-equalizing,charge-floating charge, which is adjusted by pulse width. Solar power-supply system can ensure the normal operation in the extremely severe weather (such as high temperature, low temperature and continuous 7-day rain at most).

\subsection{GPRS Wireless Data Transmission}

GPRS is the abbreviation of General Packet Radio Service; it can achieve rapid access and has such features as full use of existing network, high resource utilization rate, always on line, high transmission rate and reasonable expenses, etc[4]. This system is developed with exclusive GPRS data transmission module, which has such functions as operation mode setting, working parameters setting, data transmission and data reception, etc., and is the key function module for the realization of the wireless data transmission between collecting device and data server.

The frequently-used protocols in the GPRS data transmission are UDP protocol and TCP protocol. TCP is Transmission Control Protocol and provides connection-oriented and reliable byte stream service. TCP can ensure the reliable transmission of data by means of such functions as timeout retransmission, repeating data abandoning, data and controlling flow inspecting, etc. UDP is User Datagram Protocol, a simple datagram-oriented transport layer protocol, which doesn't establish connection before data transmission and has no functions like time-out retransmission, etc. Therefore, its transmission speed is high, but it can't ensure the reliability of data transmission[5,7].Obviously, compared with UDP, TCP has a complicated transmission and low speed. This system adopts UDP protocol to conduct data transmission and support the server's remote single station or multi-station data supplementary transmission if any soil moisture data is lost in transmission.

\section{Software System Design}

The software of DZN2 automatic soil moisture observation system is composed of data receiving and processing software and business application software. Data receiving and processing software is installed on the data server and includes data receiving and processing system, message ftp transmission system and soil moisture database. Business application software is installed on the terminal computers used by business personnel and service staff, and includes soil moisture data query and analysis system and soil moisture equipment monitoring platform.

\subsection{Data Receiving \& Processing System}

\subsubsection{Data Receiving \& Processing Software}

GPRS data receiving program receives state files and data files of collector sent through GPRS wirelessly at any time, and then the message processing program generates message files according to the requirement of "Data Transmission File Format Uploaded on Automatic Soil Moisture Observation Station" of China Meteorological Administration and saves the data of volumetric moisture content, weight moisture content, relative humidity and effective moisture storage volume, etc. of soil moisture into the database. The generated automatic soil moisture messages will be transmitted to the message server automatically through message ftp transmission system and uploaded to China Meteorological Administration regularly.

Meanwhile, GPRS data receiving program can monitor whether the hardware equipment is on line or not, how is GPRS signal strength and whether data is uploaded, etc. and the program can set the parameters of collector and sensor remotely and retransmit the missing data of stations.

\subsubsection{Soil Moisture Database}

The central station Server is established with automatic soil moisture information database and station parameter database. The information of station number, station name, longitude and latitude, altitude, collector address and $10 \mathrm{~cm}-100 \mathrm{~cm}$ soil hydrological parameters, etc. are saved in the databases, which enables the central station to receive the automatic soil moisture observation data normally and enter them to database.

3.2 Business Application Software 


\subsubsection{Automatic Soil Moisture Data Query and Analysis System}

Automatic soil moisture data query and analysis system can make query and analysis to various soil moisture elements such as volumetric moisture content, weight moisture content, relative humidity and effective moisture storage volume, etc. of 8 layers' (0-10、10-20、20-30、30-40、40-50、 50-60、70-80、90-100 cm) soil moisture. The software provides various view modes for each business and service user. Among the modes, soil moisture map can show the soil moisture maps of any layers from $10 \mathrm{~cm}$ to $100 \mathrm{~cm}$ of all observation stations in the selected area; multi-layer hourly graph can show the hourly average soil moisture elements of any multi-layer of a certain observation station in the selected area; multi-station hourly graph can show the hourly average soil moisture elements of a certain layer or the average layer of multiple observation stations in the selected area. Flexible setting and querying modes provide timely and reliable data and chart for agricultural meteorological service technical personnel to analyze soil moisture and make service material.

\subsubsection{Automatic Soil Moisture Monitoring Platform}

In order to maintain the normal operation of the automatic soil moisture observation system in a better way, automatic soil moisture monitoring platform is established to ensure the data reliability of automatic soil moisture observation system and the stability of operation[8,9]. Electronic records of all automatic soil moisture observation equipment are established and the parameter of sensor and collector calibration, detailed information of installation, maintenance and operation, etc. as well as the parameters of each station, soil type, soil parameters are saved in the database. The platform can clearly and timely show whether the device at each station is normal or not, etc. For fault station, the monitoring alarm module will send message automatically to the operator on duty of the station, the principle of the station, city bureau business department, etc. so as to promote the solution of the fault as soon as possible. The equipment maintenance module of the system will record this fault and the fault history can be queried.

\section{Calibration \& Business Acceptance of Automatic Soil Moisture Observation System}

Scientific and accurate calibration is the foundation to ensure the normal business operation of automatic soil moisture observation system [8]. Based on a large number of experimental studies, the mathematical model of soil moisture sensor is established, and through experimental studies on field calibration methods, laboratory calibration methods and direct contrast calibration methods, a four-step calibration method of automatic soil moisture observation instrument is gradually summarized: factory calibration, field calibration, operation calibration and business calibration. When the equipment is installed, make preliminary filed calibration to it according to the local soil type and empirical data and confirm the initial parameters of the sensor; after 6 months' stable operation of the equipment, get the operation calibration parameters of the sensor according to the parallel observation data and on the basis of the established mathematical models and realize the operation calibration; after 12 months' stable operation of the equipment, realize the final business calibration according to the parallel observation data and make the measurement accuracy of instrument reach an ideal range. At present, more than 1000 sets of DZN2 automatic soil moisture observation systems have been installed in stations all over the country, of which over 500 sets have passed the business acceptance of China Meteorological Administration and been put into business operation. Other automatic soil moisture observation stations are also performing the work of contrast observation, equipment calibration and data quality evaluation, etc.

\section{Conclusion}

Automatic soil moisture observation is the revolutionary transformation of agro-meteorological soil moisture observation and the important base for carrying out modern agro-meteorological service. Grasping the variation law of soil moisture is of great importance to agricultural production, drought monitoring and forecasting and other related ecological environment monitoring and forecasting service and theoretical researches [1]. As to automatic observation and data application of soil moisture, DZN2 automatic soil moisture observation system makes deep exploration in the 
respects of automatic collecting, processing, transmission, calibration and business application, etc. of soil moisture. The system has following features:

(1) Soil moisture sensor makes soil moisture observation on the basis of FDR (Frequency Domain Reflection) theory and can observe the four elements of volumetric moisture content, weight moisture content, relative humidity and effective moisture storage volume in eight layers. The monitoring has continuity, the data precision is high, the installation of instrument is simple and the soil structure will not be damaged.

(2) The system realizes the functions of automatic collecting, wireless transmission and automatic entry of soil moisture data, the query and analysis of soil moisture and equipment management, operation monitoring and failure alarm, etc.

(3) The calibrated DZN2 automatic soil moisture observation system can meet the needs of business service.

DZN2 automatic soil moisture observation system can collect soil moisture information quickly and accurately, which improves the effectiveness of observation, eliminates the manual reading error and reduces the labor intensity of staff at the grass-roots meteorological stations. The system is in line with the development direction of meteorological observation modernization. The technical theory adopted by this research has high generality and can be completely applied to the information monitoring process of other related fields.

\section{References}

[1] Chen Huailiang, Zhang Hongwei, Liu Ronghua, etc. Agricultural Drought Monitoring, Forecasting and Loss Assessment in China [J]. Science \& Technology Review, 2009, 27(11): 82-92.

[2] Xue Longqin, Chen Haibo, Shi Likui. Construction and Operation Management of Automatic Soil Moisture Observation Station Network in Henan Province [J]. Meteorological and Environmental Sciences, 2011, 34(4): 84-87

[3] China Meteorological Administration. Automatic Soil Moisture Observation Standard (Interim). 2010.

[4] Feng Yunmei; Shen Haiyan. Design of Remote Automatic Detecting System of Permafrost Temperature Based on GPRS [J]. Railway Computer Application, 2009, 18(4): 31-34.

[5] Shang Yingchun, Qi Hongyuan, Feng Yunmei, etc. The Design of an Automatic Detecting System of Permafrost Temperature and Data Analysis in Qinghai-Tibet Railway [J]. 2008, 5(30): 130-134.

[6] Li Nan, Liu Chengliang, Li Yanming, etc. Development of Remote Monitoring System for Soil Moisture Based on 3S Technology Alliance [J]. Transactions of the Chinese Society of Agricultural Engineering, 2010, 26(4): 169-174.

[7] Hu Wei, Hu Xinhua, Tong Jun, etc. Construction of Automatic Soil Moisture Observation Stations Network and Data Transmission [J]. Agriculture Network Information, 2010, (5): 72-74.

[8] Chen Jinhua, Yang Zaiqiang, Yang Taiming, etc. Real Time Observation and Forecasting System for Soil Moisture in Anhui Province [J]. Journal of Applied Meteorological Science 2011, 22(2): 249-256

[9] Hu Xinhua, Du Xiaoling, Quan Genyuan. Comparative Analysis of Conventional and AWS Soil Moisture Observations [J]. Meteorological Science and Technology, 2010, 38(2): 239-242 